\title{
Recent patent applications in biomarkers
}

\begin{tabular}{|c|c|c|c|c|c|}
\hline Patent number & Description & Assignee & Inventor & $\begin{array}{c}\text { Priority } \\
\text { application date }\end{array}$ & $\begin{array}{c}\text { Publication } \\
\text { date }\end{array}$ \\
\hline WO 2011035433 & $\begin{array}{l}\text { A method of screening for, diagnosing or detect- } \\
\text { ing lung cancer in a subject that involves deter- } \\
\text { mining a level of one or more biomarkers in a } \\
\text { sample from the subject and comparing the level } \\
\text { of each biomarker to that in a control. }\end{array}$ & $\begin{array}{l}\text { University Health } \\
\text { Network (Toronto, ON, } \\
\text { Canada) }\end{array}$ & $\begin{array}{l}\text { Diamandis EP, } \\
\text { Planque C }\end{array}$ & 9/23/2009 & $3 / 31 / 2011$ \\
\hline WO 2011035012 & $\begin{array}{l}\text { Determining whether a congestive heart failure } \\
\text { patient will respond to a pharmacotherapy treat- } \\
\text { ment, comprising determining the expression } \\
\text { level of at least ten selected biomarker genes } \\
\text { from a biological sample obtained from a conges- } \\
\text { tive heart failure patient. }\end{array}$ & $\begin{array}{l}\text { Regents of the University } \\
\text { of Colorado (Denver, CO, } \\
\text { USA) }\end{array}$ & Lowes BD & $9 / 16 / 2009$ & $3 / 24 / 2011$ \\
\hline WO 2011035083 & $\begin{array}{l}\text { A method of predicting which patients will } \\
\text { respond to apoptosis proteins-inhibiting com- } \\
\text { pound, involving administering apoptosis proteins } \\
\text { inhibitor compound to a patient and measuring } \\
\text { specific tumor necrosis factor levels. }\end{array}$ & $\begin{array}{l}\text { Novartis (Basel, } \\
\text { Switzerland) }\end{array}$ & $\begin{array}{l}\text { Firestone BG, Levine K, } \\
\text { Porter DA, Sullivan J, } \\
\text { Zawel L }\end{array}$ & 9/18/2009 & $3 / 24 / 2011$ \\
\hline WO 2011034596 & $\begin{array}{l}\text { A high-throughput method for quantifying the } \\
\text { staining of a biomarker in an original image of a } \\
\text { pathology sample to detect a disease condition } \\
\text { (e.g., ovarian cancer) involving biomarker seg- } \\
\text { mentation using hierarchical normalized cuts. }\end{array}$ & $\begin{array}{l}\text { Rutgers University } \\
\text { (New Brunswick, NJ, } \\
\text { USA), Chandran S, } \\
\text { Janowczyk A, } \\
\text { Madabhushi A }\end{array}$ & $\begin{array}{l}\text { Chandran S, } \\
\text { Janowczyk A, } \\
\text { Madabhushi A }\end{array}$ & 9/18/2009 & $3 / 24 / 2011$ \\
\hline WO 2011032155 & $\begin{array}{l}\text { Detection of a neurological condition (e.g., stroke) } \\
\text { comprising measuring the quantity of neuron- } \\
\text { specific biomarker in a sample, where the } \\
\text { synthesis of the biomarker is altered following an } \\
\text { injury, and detecting the condition based on a } \\
\text { ratio of the quantity of the biomarker in the bio- } \\
\text { logical sample. }\end{array}$ & $\begin{array}{l}\text { Banyan Biomarkers } \\
\text { (Alachua, FL, USA) }\end{array}$ & $\begin{array}{l}\text { Hayes RL, Liu MC, } \\
\text { Wang KK, Zhang Z }\end{array}$ & 9/14/2009 & $3 / 17 / 2011$ \\
\hline DE 202010009743 & $\begin{array}{l}\text { Biomarker for determining fatty acid chromatog- } \\
\text { raphy, spectroscopy, chemical analysis, enzymatic } \\
\text { or immunological diagnosis, or total fat content or } \\
\text { content of synthesized fatty acids used over hair } \\
\text { components. }\end{array}$ & $\begin{array}{l}\text { Agricon } \\
\text { (Rostock, Germany) }\end{array}$ & - & $7 / 1 / 2010$ & $3 / 17 / 2011$ \\
\hline WO 2011031344 & $\begin{array}{l}\text { Diagnosing whether an individual does or does } \\
\text { not have cancer, comprising detecting biomarker } \\
\text { values in a biological sample (e.g., serum) and } \\
\text { classifying the individual as having or not having } \\
\text { cancer based on biomarker values. }\end{array}$ & $\begin{array}{l}\text { SomaLogic } \\
\text { (Boulder, CO, USA) }\end{array}$ & $\begin{array}{l}\text { Brody EN, Gold L, } \\
\text { Mehan M, } \\
\text { Messenbaugh M, } \\
\text { Nikrad M, Ostroff RM, } \\
\text { Schwartz RS, } \\
\text { Stewart AAE, Walker J, } \\
\text { Williams SA, Zichi D }\end{array}$ & 9/9/2009 & $3 / 17 / 2011$ \\
\hline WO 2011032109 & $\begin{array}{l}\text { Predicting a level of severity or disease } \\
\text { progression of spinal muscular atrophy in a } \\
\text { patient, involving measuring levels or ratio of } \\
\text { specific markers in a biological sample and } \\
\text { comparing the measured levels or ratio to } \\
\text { reference values. }\end{array}$ & $\begin{array}{l}\text { SMA Foundation } \\
\text { (New York) }\end{array}$ & $\begin{array}{l}\text { Chen K, Forrest S, } \\
\text { Joyce C, Kobayshi D }\end{array}$ & $9 / 11 / 2009$ & $3 / 17 / 2011$ \\
\hline WO 2011028660 & $\begin{array}{l}\text { A biomarker of decitabine-sensitive cancer stem } \\
\text { cells comprising DNA methyltransferase 3B; } \\
\text { useful for treating chemotherapeutic drug- } \\
\text { resistant cancer. }\end{array}$ & $\begin{array}{l}\text { Dartmouth College } \\
\text { (Hanover, NH, USA) }\end{array}$ & $\begin{array}{l}\text { Beyrouthy MJ, } \\
\text { Spinella M }\end{array}$ & $9 / 1 / 2009$ & $3 / 10 / 2011$ \\
\hline $\begin{array}{l}\text { US } 20110024630 \text {, } \\
\text { WO } 2011016890\end{array}$ & $\begin{array}{l}\text { A device for monitoring infrared observable } \\
\text { changes in live cells in real time, comprising a } \\
\text { growth-supporting environment that defines an } \\
\text { enclosure vessel that includes an attenuated total } \\
\text { reflectance substrate comprised of a preselected } \\
\text { material. }\end{array}$ & $\begin{array}{l}\text { Battelle Memorial } \\
\text { Institute (Richland, WA, } \\
\text { USA) }\end{array}$ & $\begin{array}{l}\text { Addleman RS, } \\
\text { Riley BJ, Sacksteder CA, } \\
\text { Sundaram SK, Weber TJ }\end{array}$ & $7 / 29 / 2009$ & $\begin{array}{l}\text { 2/3/2011, } \\
\text { 2/10/2011 }\end{array}$ \\
\hline
\end{tabular}

Source: Thomson Scientific Search Service. The status of each application is slightly different from country to country. For further details, contact Thomson Scientific, 1800 Diagonal Road, Suite 250, Alexandria, Virginia 22314, USA. Tel: 1 (800) 337-9368 (http://www.thomson.com/scientific). 